\title{
Article \\ Hematological and Biochemical Reference Ranges for the Population with Sickle Cell Disease at Steady State in Tanzania
}

\author{
Anna Daniel Fome ${ }^{1,2, *}$, Raphael Z. Sangeda ${ }^{1,3}{ }^{\mathbb{D}}$, Emmanuel Balandya ${ }^{1}{ }^{\mathbb{C}}$, Josephine Mgaya ${ }^{1}$, \\ Deogratius Soka ${ }^{1}$, Furahini Tluway ${ }^{4}$, Upendo Masamu ${ }^{1}$, Siana Nkya ${ }^{1}$, Julie Makani ${ }^{1,5}$ and Bruno P. Mmbando ${ }^{1,6}$
}

Citation: Fome, A.D.; Sangeda, R.Z.; Balandya, E.; Mgaya, J.; Soka, D.; Tluway, F.; Masamu, U.; Nkya, S.; Makani, J.; Mmbando, B.P. Hematological and Biochemical Reference Ranges for the Population with Sickle Cell Disease at Steady State in Tanzania. Hemato 2022, 3 , 82-97. https://doi.org/10.3390/ hemato3010007

Academic Editor: Emmanuel Andrès

Received: 9 December 2021 Accepted: 12 January 2022 Published: 15 January 2022

Publisher's Note: MDPI stays neutral with regard to jurisdictional claims in published maps and institutional affiliations.

Copyright: (C) 2022 by the authors. Licensee MDPI, Basel, Switzerland. This article is an open access article distributed under the terms and conditions of the Creative Commons Attribution (CC BY) license (https:// creativecommons.org/licenses/by/ $4.0 /)$.
1 Muhimbili Sickle Cell Programme, Muhimbili University of Health and Allied Sciences, Dar es Salaam P.O. Box 65001, Tanzania; sangeda@gmail.com (R.Z.S.); ebalandya@yahoo.com (E.B.); jmgaya@blood.ac.tz (J.M.); zemkombozi@gmail.com (D.S.); umasamu@blood.ac.tz (U.M.); snkyamtatiro@gmail.com (S.N.); jmakani@blood.ac.tz (J.M.); b.mmbando@yahoo.com (B.P.M.)

2 Department of Economics, Mathematics and Statistics, Jordan University College, Morogoro P.O. Box 1878, Tanzania

3 Department of Pharmaceutical Microbiology, Muhimbili University of Health and Allied Sciences, Dar es Salaam P.O. Box 65013, Tanzania

4 Faculty of Health Science, Sydney Brenner Institute for Molecular Bioscience, Witwatersrand University, Johannesburg 2193, South Africa; tlywayfd@gmail.com

5 Department of Hematology and Blood Transfusion, Muhimbili University of Health and Allied Sciences, Dar es Salaam P.O. Box 65001, Tanzania

6 Tanga Centre, National Institute for Medical Research, Tanga P.O. Box 5004, Tanzania

* Correspondence: fomeannie@gmail.com

\begin{abstract}
Hematological and biochemical reference values in sickle cell disease (SCD) are crucial for patient management and the evaluation of interventions. This study was conducted at Muhimbili National Hospital (MNH) in Dar es Salaam, Tanzania, to establish laboratory reference ranges among children and adults with SCD at steady state. Patients were grouped into five age groups and according to their sex. Aggregate functions were used to handle repeated measurements within the individual level in each age group. A nonparametric approach was used to smooth the curves, and a parametric approach was used to determine SCD normal ranges. Comparison between males and females and against the general population was documented. Data from 4422 patients collected from 2004-2015 were analyzed. The majority of the patients (35.41\%) were children aged between $5-11$ years. There were no significant differences $(p \geq 0.05)$ in mean corpuscular hemoglobin concentration (MCHC), lymphocytes, basophils, and direct bilirubin observed between males and females. Significant differences $(p<0.05)$ were observed in all selected parameters across age groups except with neutrophils and MCHC in adults, as well as platelets and alkaline phosphatase in infants when the SCD estimates were compared to the general population. The laboratory reference ranges in SCD at steady state were different from those of the general population and varied with sex and age. The established reference ranges for SCD at steady state will be helpful in the management and monitoring of the progress of SCD.
\end{abstract}

Keywords: hematological parameters; biochemical parameters; reference ranges; sickle cell disease; Tanzania; steady state

\section{Introduction}

Sickle cell disease (SCD) is the most common genetic disorder globally, with an estimated 330,000 babies born with the condition annually [1]. Sub-Saharan Africa is disproportionately overburdened with SCD, where currently $75 \%$ of all patients reside in the region, and this amount is estimated to increase to $85 \%$ by the year 2050 [2]. In the absence of optimal care, up to $80 \%$ of children with SCD may die before reaching adulthood [3-5]. In high-income countries, effective management of SCD has been achieved through public health education, early diagnosis (including newborn screening), and 
therapeutic intervention to prevent or treat infections, anemia, and other sickling crises [5]. Curative options, such as bone marrow transplantation, are becoming increasingly available although challenged by the high risk of complications with the procedure and, in lowincome countries, high cost, lack of expertise, and lack of facilities [6,7].

SCD primarily affects red blood cells but also causes changes in other hematological components such as white blood cells and platelets [8,9]. Individuals with SCD go through alternating periods of stable health (steady state) and crises associated with complications such as anemia and pain [10]. These are generally associated with alteration in the quantity and function of the cellular elements in the blood. Significant alterations in hematological parameters occur in severe health conditions, such as chronic pains, and may predict prognosis [11]. While a low neutrophil count has been associated with a good prognosis in SCD patients, this is generally rare among patients with SCD at steady state [8]. Furthermore, research has shown that patients with a high leukocyte count, low steady state hemoglobin, and lower fetal hemoglobin level $(\mathrm{HbF})$ have increased risks for sickle cell crises, including stroke [12].

Overall, most of the hematological and biochemical parameters in individuals with SCD are different from those of the general population $[8,9,13,14]$. Thus, the significance of laboratory reference values for SCD has received considerable attention in recent years $[8,9,13-15]$. Unfortunately, there is a scarcity of reliable data to serve as reference values for hematological and biochemical parameters among patients with SCD in most African countries [16-18]. Only a few laboratories have their reference interval established from their population. Most other laboratories rely on reference values created many years ago and those generated from a different population whose characteristics are likely to be very different [19]. Since laboratory reference values are critical in medical decision-making, it is imperative to understand the reference values for individuals with SCD at steady state in Sub-Saharan Africa. In this study, we aimed to characterize the hematological and biochemical parameters among children and adult patients with SCD at steady state that are essential in monitoring the progress of patients under different interventions.

\section{Results}

\subsection{Demographic Characteristics}

A total of 4422 patients with SCD were included in this study. The male-to-female ratio was 1:1. The majority of patients $(1566,35.41 \%)$ were children aged between $5-11$ years, while a minority $(135,3.05 \%)$ were infants. Overall, the females were slightly older than the males $(11.59$ vs. 10.30 years; $p<0.05)$. The mean difference across the age groups when compared by sex was not significant except in the age group of 1-4 years, as presented in Table 1.

Table 1. Demographic parameters of SCD patients. Mean values were obtained after finding the mean ages within the individual level for each group.

\begin{tabular}{ccccc}
\hline Parameters & Overall & Females & Males & $p$-Values * \\
\hline \multicolumn{5}{c}{ Infants (<1 year) } \\
\hline Frequency (\%) & $135(3.05)$ & $59(1.33)$ & $76(1.72)$ & 0.426 \\
\hline Mean age \pm SD & $0.71 \pm 0.23$ & $0.69 \pm 0.26$ & $0.72 \pm 0.21$ & 0.023 \\
\hline \multicolumn{5}{c}{ Children (1-4 years) } \\
\hline Frequency (\%) & $998(22.6)$ & $478(10.80)$ & $520(11.75)$ & \\
\hline Mean age \pm SD & $3.3 \pm 1.02$ & $3.39 \pm 1.03$ & $3.25 \pm 1.00$ & \\
\hline Frequency $(\%)$ & $1566(35.41)$ & $766(17.32)$ & $800(18.09)$ & 0.144 \\
\hline Mean age \pm SD & $8.36 \pm 1.90$ & $8.44 \pm 1.90$ & $8.29 \pm 1.89$ & \\
\hline
\end{tabular}


Table 1. Cont.

\begin{tabular}{ccccc}
\hline Parameters & Overall & Females & Males & $p$-Values * \\
\hline \multicolumn{5}{c}{ Adolescents (12-17 years) } \\
\hline Frequency (\%) & $956(21.61)$ & $476(10.76)$ & $480(10.85)$ & 0.125 \\
\hline Mean age \pm SD & $14.66 \pm 1.57$ & $14.74 \pm 1.59$ & $14.59 \pm 1.55$ & 0.112 \\
\hline \multicolumn{5}{c}{ Adults $(\geq \mathbf{1 8}$ years) } \\
\hline Frequency & $767(17.34)$ & $456(10.31)$ & $311(7.03)$ \\
\hline Mean age \pm SD & $23.36 \pm 5.22$ & $23.61 \pm 5.28$ & $23.0 \pm 5.11$ \\
\hline Frequency (\%) & $4422(100.0)$ & $2235(50.54)$ & $2187(49.46)$ \\
\hline Mean age \pm SD & $10.95 \pm 7.42$ & $11.59 \pm 7.75$ & $10.30 \pm 7.01$ & $<0.05$ \\
\hline * Welch's two-sample $t$-test for the mean difference.
\end{tabular}

\subsection{Comparison of Haematological Parameters}

The mean values of hematological indices are shown in Table 2. The levels of hemoglobin and $\mathrm{MCH}$ were significantly different in all age groups except in infants, where the results were analyzed by sex. The mean $\mathrm{HbF}$ was higher in females, but the difference was statistically significant in children aged 5-11 years, adolescents, and adults ( $p$-values of $0.0006,0.0341$, and 0.0005 , respectively). While the MCHC increased slightly with age, the mean levels were comparable between females and males. The RBC and reticulocyte counts were significantly higher in males than females among patients aged $\geq 18$ years, with $p$-values of $<0.0001$ and 0.0411 or 0.0276 , respectively. The mean MCV and RDW in the infant groups were similar when the results were examined by sex. The mean platelet counts were lower in females, but the difference was only evident in children aged $1-4$ years $(p=0.0183)$. The MPV was significantly higher in female children aged $5-11$ years compared to males $(p=0.0002)$. Furthermore, the mean WBC was higher in males. However, the observed difference was not significant among infants ( $<1$ year) and adults ( $\geq 18$ years). WBC components, i.e., neutrophils and eosinophils, were evidently different in adolescents and adults when their mean levels were analyzed by sex. The mean lymphocytes (percentage) were higher in females, although significantly so only in the age groups of $<1$ and 5-11 years. On the other hand, the absolute lymphocytes were significantly higher in male children aged between 5 and 11 years and adolescents (12-17 years), with $p=0.0128$ and 0.0468 , respectively. Monocyte amounts were more elevated in males, but the difference was not statistically significant in infants and children aged 5-11 years. The mean basophil counts were comparable between males and females.

Figure 1 represents the smoothed mean curves of the hematological parameters, which were statistically different between males and females. In the figure, the bandwidth represents a $95 \%$ confidence interval. Therefore, there is a 0.95 probability that the true regression line for the population lies within the confidence interval. 

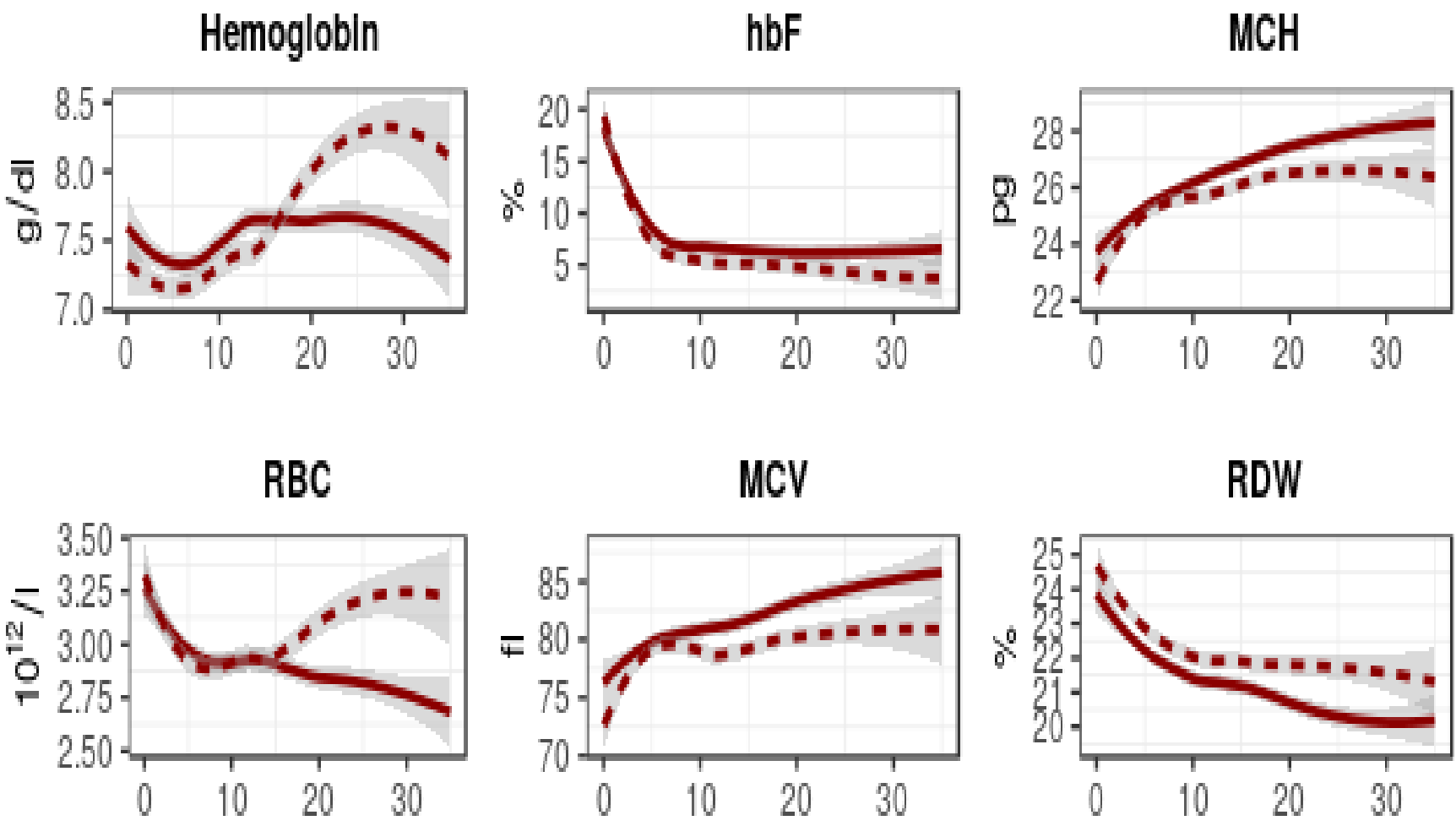

RDW

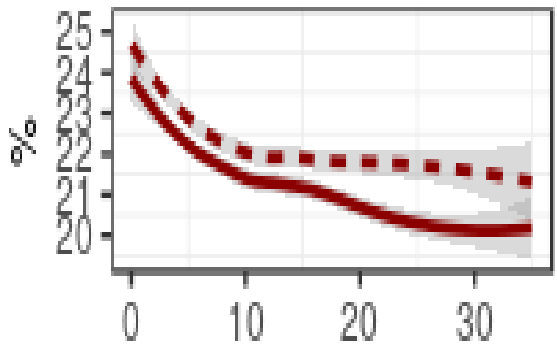

Platelet
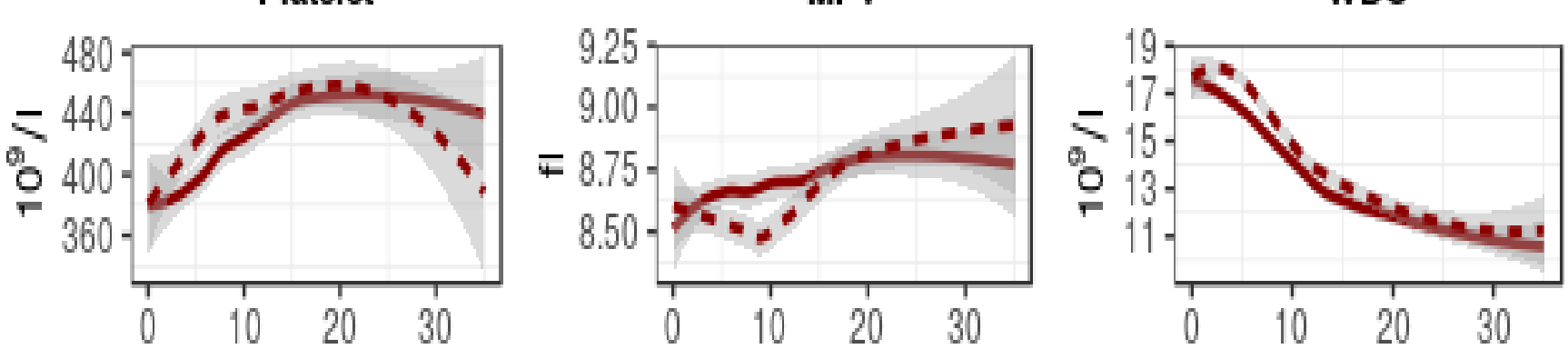

Neutrophils

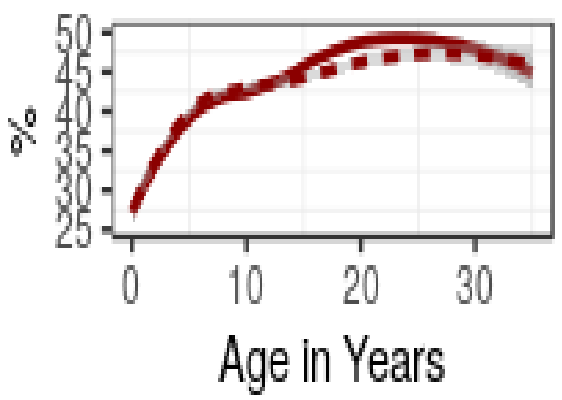

Eosinophils

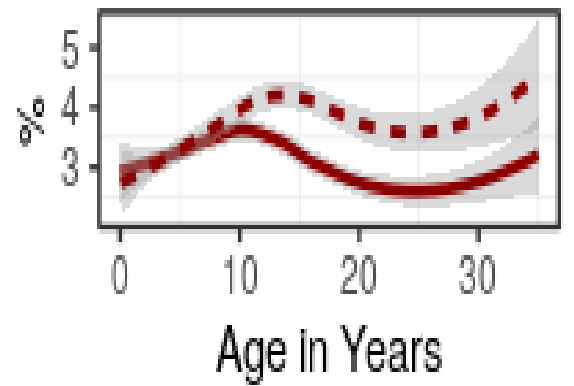

Monocytes

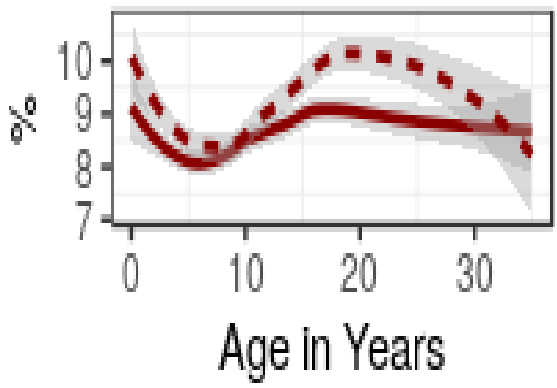

Figure 1. Smoothed mean curves for selected hematological parameters by sex. Solid lines denote the mean values for females and dashed lines denote the mean values for males. 
Table 2. Steady state hematological reference values calculated as mean (mean $\pm 1.96 \mathrm{SD}$ ) in SCD, categorized by age groups and sex.

\begin{tabular}{|c|c|c|c|c|c|c|c|}
\hline \multirow[b]{2}{*}{$\begin{array}{c}\text { Age Group } \\
\text { (Years) }\end{array}$} & \multicolumn{2}{|r|}{ Females } & \multicolumn{2}{|r|}{ Males } & \multicolumn{2}{|r|}{ Combined } & \multirow[b]{2}{*}{$p$-Values * } \\
\hline & $\mathbf{n}$ & $\begin{array}{c}\text { Mean } \\
(\text { Mean } \pm 1.96 S D)\end{array}$ & $\mathbf{n}$ & $\begin{array}{c}\text { Mean } \\
(\text { Mean } \pm 1.96 S D)\end{array}$ & $\mathbf{n}$ & $\begin{array}{c}\text { Mean } \\
(\text { Mean } \pm 1.96 S D)\end{array}$ & \\
\hline \multicolumn{8}{|c|}{ Hemoglobin $(\mathrm{g} / \mathrm{dL})$} \\
\hline$<1$ & 59 & $7.3(4.8-9.8)$ & 76 & $7.2(4.5-9.8)$ & 135 & $7.2(4.6-9.8)$ & 0.4574 \\
\hline $1-4$ & 477 & $7.4(5.0-9.7)$ & 518 & $7.2(4.7-9.6)$ & 995 & $7.3(4.9-9.7)$ & 0.0128 \\
\hline $5-11$ & 766 & $7.4(5.1-9.6)$ & 799 & $7.2(5.1-9.3)$ & 1565 & $7.3(5.1-9.5)$ & 0.0018 \\
\hline $12-17$ & 476 & $7.6(5.4-9.7)$ & 480 & $7.4(5.1-9.7)$ & 956 & $7.5(5.3-9.7)$ & 0.0171 \\
\hline$\geq 18$ & 456 & $7.6(5.1-10.0)$ & 310 & $8.2(5.0-11.3)$ & 766 & $7.8(5.0-10.6)$ & $<0.0001$ \\
\hline \multicolumn{8}{|c|}{ HbF (\%) } \\
\hline$<1$ & 29 & $16.5(4.0-67.7)$ & 42 & $16.0(3.1-81.2)$ & 71 & $16.2(3.5-75.2)$ & 0.7882 \\
\hline $1-4$ & 278 & $9.8(2.7-35.4)$ & 272 & $9.3(2.9-29.5)$ & 550 & $9.6(2.8-32.4)$ & 0.1696 \\
\hline $5-11$ & 379 & $6.6(1.9-23.1)$ & 377 & $5.5(1.5-19.9)$ & 756 & $6.0(1.6-21.8)$ & 0.0006 \\
\hline $12-17$ & 150 & $5.8(1.3-24.4)$ & 135 & $4.9(1.3-18.0)$ & 285 & $5.3(1.3-21.4)$ & 0.0341 \\
\hline$\geq 18$ & 162 & $5.9(1.5-22.5)$ & 103 & $3.9(0.9-16.6)$ & 265 & $5.0(1.2-21.1)$ & 0.0005 \\
\hline \multicolumn{8}{|c|}{ MCH (pg) } \\
\hline$<1$ & 58 & $23.4(17.4-29.4)$ & 75 & $22.4(14.9-30.0)$ & 133 & $22.9(15.9-29.8)$ & 0.1109 \\
\hline $1-4$ & 476 & $25.0(18.7-31.3)$ & 514 & $24.5(18.2-30.9)$ & 990 & $24.8(18.4-31.1)$ & 0.0357 \\
\hline $5-11$ & 762 & $25.8(19.0-32.6)$ & 798 & $25.4(18.8-32.1)$ & 1560 & $25.6(18.9-32.4)$ & 0.0392 \\
\hline $12-17$ & 476 & $26.8(20.2-33.4)$ & 478 & $25.9(19.2-32.7)$ & 954 & $26.4(19.6-33.1)$ & $<0.0001$ \\
\hline$\geq 18$ & 453 & $27.6(20.3-34.9)$ & 311 & $26.5(19.6-33.5)$ & 764 & $27.2(20.0-34.4)$ & $<0.0001$ \\
\hline \multicolumn{8}{|c|}{$\operatorname{MCHC}(\mathrm{g} / \mathrm{dL})$} \\
\hline$<<1$ & 58 & $31.0(28.6-33.5)$ & 76 & $30.9(27.4-34.4)$ & 134 & $31.0(27.9-34.0)$ & 0.5994 \\
\hline $1-4$ & 477 & $31.4(28.1-34.7)$ & 518 & $31.4(28.0-34.9)$ & 995 & $31.4(28.0-34.8)$ & 0.9934 \\
\hline $5-11$ & 765 & $32.1(28.9-35.4)$ & 799 & $32.1(28.6-35.6)$ & 1564 & $32.1(28.7-35.5)$ & 0.8559 \\
\hline $12-17$ & 476 & $32.8(29.5-36.1)$ & 478 & $32.9(29.6-36.1)$ & 954 & $32.8(29.6-36.1)$ & 0.5454 \\
\hline$\geq 18$ & 456 & $32.9(29.8-35.9)$ & 311 & $32.9(29.7-36.1)$ & 767 & $32.9(29.8-36.0)$ & 0.9422 \\
\hline \multicolumn{8}{|c|}{ RBC Count $\left(\times 10^{12} / \mathrm{L}\right)$} \\
\hline$<1$ & 59 & $3.2(1.7-4.6)$ & 76 & $3.3(1.4-5.2)$ & 135 & $3.2(1.5-4.9)$ & 0.4144 \\
\hline $1-4$ & 478 & $3.0(1.6-4.4)$ & 518 & $3.0(1.5-4.4)$ & 996 & $3.0(1.6-4.4)$ & 0.3762 \\
\hline $5-11$ & 766 & $2.9(1.6-4.2)$ & 800 & $2.8(1.5-4.2)$ & 1566 & $2.9(1.6-4.2)$ & 0.2956 \\
\hline $12-17$ & 476 & $2.8(1.7-4.0)$ & 478 & $2.9(1.5-4.3)$ & 954 & $2.9(1.6-4.2)$ & 0.2519 \\
\hline$\geq 18$ & 456 & $2.8(1.5-4.1)$ & 310 & $3.1(1.4-4.9)$ & 766 & $2.9(1.4-4.5)$ & $<0.0001$ \\
\hline \multicolumn{8}{|c|}{ MCV (fL) } \\
\hline$<1$ & 58 & $75.4(57.8-92.9)$ & 76 & $72.7(50.9-94.4)$ & 134 & $73.8(53.7-94.0)$ & 0.2167 \\
\hline $1-4$ & 477 & $79.4(61.2-97.7)$ & 517 & $78.1(59.9-96.2)$ & 994 & $78.7(60.5-97.0)$ & 0.0106 \\
\hline $5-11$ & 765 & $80.3(60.7-99.9)$ & 799 & $79.2(60.7-97.7)$ & 1564 & $79.7(60.7-98.8)$ & 0.0140 \\
\hline $12-17$ & 476 & $81.8(64.0-99.5$ & 478 & $78.9(60.2-97.5)$ & 954 & 80.3 (61.9-98.8) & $<0.0001$ \\
\hline$\geq 18$ & 455 & $83.9(64.1-103.7)$ & 311 & $80.7(61.5-99.9)$ & 766 & $82.6(62.8-102.4)$ & $<0.0001$ \\
\hline \multicolumn{8}{|c|}{ RDW (\%) } \\
\hline$<1$ & 59 & $23.7(16.9-30.5)$ & 75 & $24.3(17.4-31.3)$ & 134 & $24.1(17.2-30.9)$ & 0.1860 \\
\hline $1-4$ & 476 & $22.6(16.2-28.9)$ & 518 & $23.3(16.9-29.7)$ & 994 & $22.9(16.5-29.4)$ & $<0.0001$ \\
\hline $5-11$ & 764 & $21.6(15.4-27.8)$ & 800 & $22.3(16.3-28.4)$ & 1564 & $22.0(15.8-28.1)$ & $<0.0001$ \\
\hline $12-17$ & 476 & $21.2(15.0-27.5)$ & 478 & $21.9(16.1-27.6)$ & 956 & $21.6(15.5-27.6)$ & $<0.0001$ \\
\hline$\geq 18$ & 456 & $20.3(14.5-26.1)$ & 310 & $21.6(15.6-27.6)$ & 766 & $20.8(14.8-26.8)$ & $<0.0001$ \\
\hline \multicolumn{8}{|c|}{ Reticulocyte (\%) } \\
\hline$<1$ & 20 & $12.1(3.0-27.2)$ & 17 & $13.0(3.2-29.4)$ & 37 & $12.5(3.2-28.0)$ & 0.7032 \\
\hline $1-4$ & 201 & $13.4(3.6-29.3)$ & 221 & $12.5(2.0-31.9)$ & 422 & $12.9(2.7-30.7)$ & 0.4093 \\
\hline $5-11$ & 336 & $12.4(3.8-26.0)$ & 365 & $12.1(3.6-25.6)$ & 701 & $12.3(3.7-25.8)$ & 0.9524 \\
\hline $12-17$ & 235 & $12.0(3.8-24.7)$ & 198 & $11.1(3.4-23.3)$ & 433 & $11.6(3.6-24.1)$ & 0.0894 \\
\hline$\geq 18$ & 202 & $11.9(3.9-24.2)$ & 122 & $11.0(3.9-21.8)$ & 324 & $11.6(3.9-23.4)$ & 0.0411 \\
\hline
\end{tabular}


Table 2. Cont.

\begin{tabular}{|c|c|c|c|c|c|c|c|}
\hline \multirow[b]{2}{*}{$\begin{array}{l}\text { Age Group } \\
\text { (Years) }\end{array}$} & \multicolumn{2}{|r|}{ Females } & \multicolumn{2}{|r|}{ Males } & \multicolumn{2}{|r|}{ Combined } & \multirow[b]{2}{*}{$p$-Values * } \\
\hline & $\mathbf{n}$ & $\begin{array}{c}\text { Mean } \\
(\text { Mean } \pm 1.96 S D)\end{array}$ & $\mathbf{n}$ & $\begin{array}{c}\text { Mean } \\
(\text { Mean } \pm \text { 1.96SD) }\end{array}$ & $\mathbf{n}$ & $\begin{array}{c}\text { Mean } \\
(\text { Mean } \pm 1.96 S D)\end{array}$ & \\
\hline \multicolumn{8}{|c|}{ Reticulocyte Count $\left(\times 10^{12} / \mathrm{L}\right)$} \\
\hline$<1$ & 20 & $0.38(0.07-0.70)$ & 17 & $0.42(0.12-0.72)$ & 37 & $0.40(0.09-0.71)$ & 0.4462 \\
\hline $1-4$ & 201 & $0.42(0.04-0.79)$ & 221 & $0.39(0.01-0.78)$ & 422 & $0.40(0.02-0.79)$ & 0.1977 \\
\hline $5-11$ & 336 & $0.37(0.04-0.70)$ & 365 & $0.37(0.06-0.68)$ & 701 & $0.37(0.05-0.69)$ & 0.7346 \\
\hline $12-17$ & 235 & $0.36(0.07-0.66)$ & 198 & $0.33(0.66-0.61)$ & 433 & $0.35(0.06-0.64)$ & 0.0371 \\
\hline$\geq 18$ & 202 & $0.34(0.07-0.61)$ & 122 & $0.38(0.09-0.66)$ & 324 & $0.36(0.08-0.63)$ & 0.0276 \\
\hline \multicolumn{8}{|c|}{ Platelet Count $\left(\times 10^{9} / \mathrm{L}\right)$} \\
\hline$<1$ & 59 & $397.9(73.3-722.4)$ & 76 & $410.8(56.8-764.7)$ & 135 & $405.1(64.8-745.5)$ & 0.7935 \\
\hline $1-4$ & 476 & $375.1(55.8-694.4)$ & 517 & $399.2(67.0-731.4)$ & 993 & $387.7(60.9-714.4)$ & 0.0183 \\
\hline $5-11$ & 766 & $422.3(110.9-733.6)$ & 798 & $440.2(103.8-776.6)$ & 1564 & $431.4(106.7-756.2)$ & 0.1210 \\
\hline $12-17$ & 476 & $447.0(130.6-763.4)$ & 477 & $453.3(142.5-764.1)$ & 953 & $450.2(136.7-763.7)$ & 0.9320 \\
\hline$\geq 18$ & 456 & 442.9 (149.5-736.3) & 309 & $447.0(149.2-744.9)$ & 765 & $444.6(149.6-739.6)$ & 0.7580 \\
\hline \multicolumn{8}{|c|}{ MPV (fL) } \\
\hline$<1$ & 53 & $8.5(6.8-9.9)$ & 68 & $8.3(6.4-10.5)$ & 121 & $8.4(6.5-10.2)$ & 0.9604 \\
\hline $1-4$ & 458 & $8.7(6.9-10.3)$ & 498 & $8.6(6.8-10.1)$ & 956 & $8.6(6.9-10.2)$ & 0.2977 \\
\hline $5-11$ & 741 & $8.6(7.0-10.2)$ & 781 & $8.4(6.8-10.1)$ & 1522 & $8.6(6.9-10.2)$ & 0.0002 \\
\hline $12-17$ & 467 & $8.5(6.9-10.4)$ & 470 & $8.5(7.0-10.3)$ & 937 & $8.5(7.0-10.4)$ & 0.7275 \\
\hline$\geq 18$ & 443 & $8.5(7.1-10.5)$ & 304 & $8.6(7.1-10.6)$ & 747 & $8.6(7.1-10.5)$ & 0.3750 \\
\hline \multicolumn{8}{|c|}{ WBC $\left(\times 10^{9} / \mathrm{L}\right)$} \\
\hline$<1$ & 57 & $16.4(3.8-29.1)$ & 72 & $16.6(1.3-31.3)$ & 129 & $16.4(2.4-30.3)$ & 0.4381 \\
\hline $1-4$ & 473 & $17.1(6.5-27.6)$ & 518 & $18.2(7.3-29.2)$ & 991 & 17.7 (6.9-28.5) & 0.0006 \\
\hline $5-11$ & 798 & $14.7(5.6-23.8)$ & 766 & $15.7(7.1-24.4)$ & 1564 & $15.2(6.3-24.1)$ & $<0.0001$ \\
\hline $12-17$ & 476 & $12.5(5.8-19.2)$ & 477 & $13.3(6.0-20.7)$ & 953 & $12.9(5.9-20.0)$ & 0.0003 \\
\hline$\geq 18$ & 455 & $11.6(4.7-18.4)$ & 311 & $11.9(4.3-19.6)$ & 766 & $11.7(4.5-18.9)$ & 0.4009 \\
\hline \multicolumn{8}{|c|}{ Neutrophils (\%) } \\
\hline$<1$ & 56 & $27.2(7.0-47.3)$ & 69 & $29.3(8.2-50.4)$ & 125 & $28.3(7.6-49.1)$ & 0.1985 \\
\hline $1-4$ & 447 & $36.5(18.1-55.0)$ & 482 & $36.7(18.1-55.2)$ & 929 & $36.6(18.1-55.1)$ & 0.9277 \\
\hline $5-11$ & 724 & $41.6(25.2-58.0)$ & 783 & $42.2(25.4-58.9)$ & 1507 & $41.9(25.3-58.5)$ & 0.2629 \\
\hline $12-17$ & 465 & $45.5(29.1-62.0)$ & 460 & $44.0(27.6-60.5)$ & 925 & $44.8(28.3-61.3)$ & 0.0091 \\
\hline$\geq 18$ & 449 & $48.5(28.9-68.0)$ & 305 & $46.9(25.5-68.1)$ & 754 & $47.8(27.6-68.1)$ & 0.0132 \\
\hline \multicolumn{8}{|c|}{ Neutrophils Count $\left(\times 10^{9} / \mathrm{L}\right)$} \\
\hline$<1$ & 56 & $4.3(0.02-8.6)$ & 69 & $4.7(-0.9-10.4)$ & 125 & $4.5(-0.5-9.6)$ & 0.3250 \\
\hline $1-4$ & 447 & $6.1(1.6-10.6)$ & 482 & $6.5(2.1-10.8)$ & 929 & $6.3(1.8-10.7)$ & 0.0087 \\
\hline $5-11$ & 724 & $6.0(1.8-10.2)$ & 783 & $6.6(2.2-10.9$ & 1507 & $6.3(2.0-10.6)$ & $<0.0001$ \\
\hline $12-17$ & 465 & $5.7(1.9-9.5)$ & 460 & $5.8(1.7-9.9)$ & 925 & $5.8(1.8-9.7)$ & 0.1927 \\
\hline$\geq 18$ & 449 & $5.6(1.1-10.2)$ & 305 & $5.5(1.0-10.1)$ & 754 & $5.6(1.1-10.2)$ & 0.6492 \\
\hline \multicolumn{8}{|c|}{ Lymphocytes (\%) } \\
\hline$<1$ & 59 & $56.6(29.7-83.5)$ & 75 & $53.3(29.3-77.4)$ & 134 & $54.8(29.3-80.2)$ & 0.0740 \\
\hline $1-4$ & 472 & $49.4(29.4-69.5)$ & 516 & $48.6(28.2-69.1)$ & 988 & $49.0(28.8-69.3)$ & 0.2216 \\
\hline $5-11$ & 763 & $43.9(26.7-61.1)$ & 796 & $43.0(26.2-59.8)$ & 1559 & $43.5(26.4-60.5)$ & 0.0612 \\
\hline $12-17$ & 474 & $40.1(24.1-56.1)$ & 476 & $39.6(23.6-55.7)$ & 950 & $39.9(23.9-55.9)$ & 0.3484 \\
\hline$\geq 18$ & 454 & $37.0(20.5-53.5)$ & 310 & $36.5(17.5-55.5)$ & 764 & $36.8(19.2-54.3)$ & 0.4455 \\
\hline \multicolumn{8}{|c|}{ Lymphocytes Count $\left(\times 10^{9} / \mathrm{L}\right)$} \\
\hline$<1$ & 59 & $9.3(0.04-18.7)$ & 75 & $8.7(0.0-18.2)$ & 134 & $9.0(0.0-18.4)$ & 0.4594 \\
\hline $1-4$ & 472 & $8.5(1.7-15.2)$ & 516 & $8.9(1.9-15.8)$ & 988 & $8.7(1.8-15.5)$ & 0.0758 \\
\hline $5-11$ & 763 & $6.4(1.3-11.6)$ & 796 & $6.8(1.8-11.7)$ & 1559 & $6.6(1.5-11.7)$ & 0.0128 \\
\hline $12-17$ & 474 & $5.0(1.6-8.3)$ & 476 & $5.2(2.1-8.3)$ & 950 & $5.1(1.8-8.3)$ & 0.0468 \\
\hline$\geq 18$ & 454 & $4.2(1.5-6.9)$ & 310 & $4.2(1.1-7.3)$ & 764 & $4.2(1.3-7.1)$ & 0.6696 \\
\hline
\end{tabular}


Table 2. Cont.

\begin{tabular}{|c|c|c|c|c|c|c|c|}
\hline \multirow[b]{2}{*}{$\begin{array}{c}\text { Age Group } \\
\text { (Years) }\end{array}$} & \multicolumn{2}{|r|}{ Females } & \multicolumn{2}{|r|}{ Males } & \multicolumn{2}{|r|}{ Combined } & \multirow[b]{2}{*}{$p$-Values * } \\
\hline & $\mathbf{n}$ & $\begin{array}{c}\text { Mean } \\
(\text { Mean } \pm 1.96 S D)\end{array}$ & $\mathbf{n}$ & $\begin{array}{c}\text { Mean } \\
(\text { Mean } \pm 1.96 S D)\end{array}$ & $\mathbf{n}$ & $\begin{array}{c}\text { Mean } \\
(\text { Mean } \pm 1.96 S D)\end{array}$ & \\
\hline \multicolumn{8}{|c|}{ Monocytes (\%) } \\
\hline$<1$ & 57 & $8.8(3.3-23.4)$ & 74 & $9.7(3.7-25.7)$ & 131 & $9.3(3.5-24.7)$ & 0.1492 \\
\hline $1-4$ & 466 & $8.3(3.7-18.6)$ & 507 & $8.8(3.7-20.5)$ & 973 & $8.6(3.7-19.6)$ & 0.0252 \\
\hline $5-11$ & 756 & $8.7(4.2-18.1)$ & 793 & $8.9(4.1-19.0)$ & 1549 & $8.8(4.1-18.5)$ & 0.3807 \\
\hline $12-17$ & 473 & $9.4(4.6-19.2)$ & 472 & $9.9(4.8-20.4)$ & 945 & $9.7(4.7-19.8)$ & 0.0121 \\
\hline$\geq 18$ & 449 & $9.5(4.7-19.0)$ & 306 & $10.5(5.0-22.2)$ & 755 & $9.9(4.8-20.4)$ & $<0.0001$ \\
\hline \multicolumn{8}{|c|}{ Monocytes Count $\left(\times 10^{9} / \mathrm{L}\right)$} \\
\hline$<1$ & 57 & $1.4(0.0-7.5)$ & 74 & $1.6(0.4-15.4)$ & 131 & $2.2(0.1-12.0)$ & 0.2325 \\
\hline $1-4$ & 466 & $1.4(0.0-8.3)$ & 507 & $1.6(0.0-10.4)$ & 973 & $2.1(0.0-9.4)$ & 0.0002 \\
\hline $5-11$ & 756 & $1.2(0.0-5.9)$ & 793 & $1.3(0.2-7.4)$ & 1549 & $1.6(0.0-6.7)$ & 0.0001 \\
\hline $12-17$ & 473 & $1.1(0.0-5.0)$ & 472 & $1.2(0.03-4.2)$ & 945 & $1.4(0.01-5.3)$ & 0.0002 \\
\hline$\geq 18$ & 449 & $1.0(0.0-3.8)$ & 306 & $1.2(0.0-4.3)$ & 755 & $1.2(0.0-4.6)$ & $<0.0001$ \\
\hline \multicolumn{8}{|c|}{ Eosinophils (\%) } \\
\hline$<1$ & 59 & $3.7(1.2-11.5)$ & 72 & $3.4(1.1-10.3)$ & 131 & $3.5(1.1-10.8)$ & 0.4710 \\
\hline $1-4$ & 462 & $3.5(1.2-9.8)$ & 501 & $3.5(1.2-9.7)$ & 963 & $3.5(1.2-9.8)$ & 0.8414 \\
\hline $5-11$ & 743 & $3.8(1.3-11.4)$ & 790 & $4.0(1.4-11.5)$ & 1533 & $3.9(1.3-11.5)$ & 0.1706 \\
\hline $12-17$ & 470 & $3.6(1.3-9.9)$ & 463 & $4.5(1.5-12.8)$ & 933 & $4.0(1.4-11.5)$ & $<0.0001$ \\
\hline$\geq 18$ & 454 & $3.2(1.1-8.9)$ & 307 & $3.8(1.2-12.2)$ & 761 & $3.5(1.1-10.3)$ & $<0.0001$ \\
\hline \multicolumn{8}{|c|}{ Eosinophils Count $\left(\times 10^{9} / \mathrm{L}\right)$} \\
\hline$<1$ & 59 & $0.53(0.0-1.44)$ & 72 & $0.45(0.0-1.20)$ & 131 & $0.48(0.0-1.31)$ & 0.3034 \\
\hline $1-4$ & 462 & $0.51(0.0-1.34)$ & 501 & $0.54(0.0-1.39)$ & 963 & $0.53(0.0-1.37)$ & 0.2644 \\
\hline $5-11$ & 743 & $0.51(0.0-1.53)$ & 790 & $0.58(0.0-1.65)$ & 1533 & $0.55(0.0-1.59)$ & 0.0078 \\
\hline $12-17$ & 470 & $0.40(0.0-1.17)$ & 463 & $0.57(0.0-1.69)$ & 933 & $0.48(0.0-1.46)$ & $<0.0001$ \\
\hline$\geq 18$ & 454 & $0.32(0.0-0.93)$ & 307 & $0.44(0.0-1.40)$ & 761 & $0.37(0.0-1.15)$ & $<0.0001$ \\
\hline \multicolumn{8}{|c|}{ Basophils (\%) } \\
\hline$<1$ & 58 & $2.5(1.3-5.0)$ & 72 & $2.3(0.9-5.5)$ & 130 & $2.4(1.1-5.3)$ & 0.1711 \\
\hline $1-4$ & 471 & $2.1(1.2-3.8)$ & 510 & $2.1(1.2-3.8)$ & 981 & $2.1(1.2-3.8)$ & 0.6519 \\
\hline $5-11$ & 754 & $2.0(1.1-3.4)$ & 795 & $2.0(1.2-3.4)$ & 1549 & $2.0(1.1-3.4)$ & 0.9251 \\
\hline $12-17$ & 473 & $1.9(1.1-3.4)$ & 474 & $1.9(1.1-3.5)$ & 947 & $1.9(1.1-3.5)$ & 0.9398 \\
\hline$\geq 18$ & 456 & $1.9(1.2-3.0)$ & 310 & $1.9(1.1-3.4)$ & 766 & $1.9(1.1-3.1)$ & 0.2761 \\
\hline
\end{tabular}

* Welch's two-sample $t$-test. SD; standard deviation.

\subsection{Comparison of Biochemical Parameters}

The variations of the mean values of biochemical parameters between male and female cases are shown in Table 3. While the mean direct bilirubin was comparable between males and females across age groups, the total bilirubin was significantly different only in adults $(p=0.0243)$. The mean AST was significantly higher in females among infants and significantly higher in males among children aged 5-11 years and adolescents. The mean ALP and creatinine were higher in adult males compared to adult females. Females aged 12-17 years had significantly lower levels of LDH (by 75.5 U/L) compared to males.

Table 3. Steady state biochemical reference values calculated as mean (mean $\pm 1.96 \mathrm{SD}$ ) in SCD, categorized by age groups and sex.

\begin{tabular}{cccccccc}
\hline $\begin{array}{c}\text { Age Group } \\
\text { (Years) }\end{array}$ & $\mathbf{n}$ & $\begin{array}{c}\text { Females } \\
\text { (Mean } \pm \text { 1.96SD) }\end{array}$ & $\mathbf{n}$ & $\begin{array}{c}\text { Males } \\
\text { (Mean } \pm \text { 1.96SD) }\end{array}$ & $\mathbf{n}$ & $\begin{array}{c}\text { Mean } \\
\text { (Mean } \pm \text { 1.96SD) }\end{array}$ & $p$-Values * \\
\hline & \multicolumn{7}{c}{ Bilirubin direct $(\boldsymbol{\mu m o l} / \mathbf{L})$} \\
\hline 1 & 13 & $9.9(0.8-119.4)$ & 17 & $8.6(1.2-59.6)$ & 30 & $9.2(1.0-80.2)$ & 0.6752 \\
$1-4$ & 154 & $7.4(1.0-54.8)$ & 169 & $6.7(0.7-64.1)$ & 323 & $7.0(0.8-59.6)$ & 0.5992 \\
$5-11$ & 308 & $7.8(1.0-56.6)$ & 353 & $7.6(1.1-53.3)$ & 661 & $7.7(1.1-54.8)$ & 0.5685 \\
$12-17$ & 198 & $6.0(0.5-62.0)$ & 184 & $6.0(0.6-55.5)$ & 382 & $6.0(0.6-58.7)$ & 0.8477 \\
$\geq 18$ & 166 & $6.7(0.9-48.4)$ & 106 & $6.1(0.6-61.2)$ & 272 & $6.5(0.7-53.3)$ & 0.7343 \\
\hline
\end{tabular}


Table 3. Cont.

\begin{tabular}{|c|c|c|c|c|c|c|c|}
\hline \multirow{2}{*}{$\begin{array}{l}\text { Age Group } \\
\text { (Years) }\end{array}$} & \multicolumn{2}{|r|}{ Females } & \multicolumn{2}{|r|}{ Males } & \multicolumn{2}{|r|}{ Combined } & \multirow[b]{2}{*}{$p$-Values * } \\
\hline & $\mathbf{n}$ & $\begin{array}{c}\text { Mean } \\
(\text { Mean } \pm 1.96 S D)\end{array}$ & $\mathbf{n}$ & $\begin{array}{c}\text { Mean } \\
(\text { Mean } \pm 1.96 S D)\end{array}$ & $\mathbf{n}$ & $\begin{array}{c}\text { Mean } \\
(\text { Mean } \pm 1.96 S D)\end{array}$ & \\
\hline \multicolumn{8}{|c|}{ Bilirubin Total $(\mu \mathrm{mol} / \mathrm{L})$} \\
\hline$<1$ & 17 & 35.4 (10.7-117.3) & 18 & $30.2(8.2-110.8)$ & 35 & $32.6(9.4-113.1)$ & 0.7165 \\
\hline $1-4$ & 153 & 39.5 (11.4-136.8) & 158 & 36.4 (11.4-116.4) & 311 & 37.9 (11.4-126.2) & 0.2966 \\
\hline $5-11$ & 280 & $50.1(14.6-170.9)$ & 318 & 50.5 (15.4-165.9) & 598 & $50.3(15.0-168.1)$ & 0.8201 \\
\hline $12-17$ & 162 & $59.4(15.6-225.7)$ & 160 & $58.0(11.5-291.3)$ & 322 & 58.7 (13.4-257.4) & 0.8052 \\
\hline$\geq 18$ & 152 & $48.9(15.1-158.5)$ & 85 & $59.9(13.7-161.7)$ & 237 & $52.6(14.3-193.4)$ & 0.0243 \\
\hline \multicolumn{8}{|c|}{ AST (U/L) } \\
\hline$<1$ & 18 & $51.6(13.3-114.9)$ & 16 & $39.4(18.3-88.5)$ & 34 & $45.7(14.0-95.4)$ & 0.0180 \\
\hline $1-4$ & 156 & $44.8(20.1-79.4)$ & 162 & $46.2(21.2-80.7)$ & 318 & $45.5(20.7-80.0)$ & 0.2745 \\
\hline $5-11$ & 283 & $43.1(20.9-73.4)$ & 321 & $46.4(22.8-78.3)$ & 604 & $44.9(21.8-76.2)$ & 0.0029 \\
\hline $12-17$ & 165 & $40.7(16.2-76.4)$ & 161 & $45.9(19.8-82.8)$ & 326 & $43.2(17.7-80.0)$ & 0.0009 \\
\hline$\geq 18$ & 155 & $33.1(12.2-64.0)$ & 87 & $34.7(12.7-67.5)$ & 242 & $33.6(12.4-65.3)$ & 0.5586 \\
\hline \multicolumn{8}{|c|}{ ALP (IU/L) } \\
\hline$<1$ & 18 & $305.4(89.4-650.0)$ & 16 & $335.5(90.8-734.5)$ & 34 & $319.4(91.6-685.0)$ & 0.3427 \\
\hline $1-4$ & 158 & $290.2(119.0-563.7)$ & 163 & $300.1(106.5-577.8)$ & 321 & $297.6(112.7-570.6)$ & 0.8089 \\
\hline 5-11 & 285 & $235.7(86.1-458.9)$ & 322 & $240.2(107.0-426.6)$ & 607 & $238.1(96.6-442.3)$ & 0.4232 \\
\hline $12-17$ & 167 & $222.6(61.9-482.6)$ & 162 & $240.3(89.3-464.4)$ & 329 & $231.2(74.2-475.1)$ & 0.1035 \\
\hline$\geq 18$ & 154 & $125.1(30.0-285.3)$ & 89 & $158.3(35.8-367.9)$ & 243 & $136.8(30.5-319.5)$ & 0.0005 \\
\hline \multicolumn{8}{|c|}{ LDH (U/L) } \\
\hline$<1$ & 18 & $794.1(123.4-2047.8)$ & 16 & $829.4(234.0-1789.4)$ & 34 & 810.6 (174.1-1913.9) & 0.9313 \\
\hline $1-4$ & 151 & $903.0(314.8-1794.2)$ & 159 & 946.9 (352.7-1828.6) & 310 & $925.4(333.9-1812.0)$ & 0.2646 \\
\hline $5-11$ & 279 & 840.1 (316.5-1614.4) & 316 & $819.2(236.5-1752.7)$ & 595 & $829.0(271.3-1690.2)$ & 0.5434 \\
\hline $12-17$ & 166 & 784.7 (208.7-1728.8) & 159 & $860.2(282.7-1750.8)$ & 325 & $821.2(241.8-1744.1)$ & 0.0207 \\
\hline$\geq 18$ & 153 & $637.9(156.4-1444.6)$ & 87 & $714.9(131.8-1763.4)$ & 240 & $665.3(145.0-1563.8)$ & 0.2412 \\
\hline \multicolumn{8}{|c|}{ Creatinine $(\mu \mathrm{mol} / \mathrm{L})$} \\
\hline$<1$ & 18 & $31.7(6.7-75.3)$ & 18 & $32.8(17.4-52.9)$ & 36 & $32.2(10.8-65.0)$ & 0.9620 \\
\hline $1-4$ & 158 & $32.6(18.9-50.0)$ & 162 & $33.3(18.1-53.0)$ & 320 & $32.9(18.5-51.5)$ & 0.4853 \\
\hline 5-11 & 284 & $31.7(16.8-51.4)$ & 320 & $33.4(17.1-55.2)$ & 604 & $32.6(16.9-53.5)$ & 0.0547 \\
\hline $12-17$ & 167 & $35.1(18.4-62.3)$ & 168 & $37.8(16.1-63.1)$ & 335 & $36.4(17.2-62.7)$ & 0.2763 \\
\hline$\geq 18$ & 151 & $48.8(20.4-75.9)$ & 87 & $51.3(23.8-89.0)$ & 238 & $46.4(21.1-81.6)$ & 0.0002 \\
\hline
\end{tabular}

* Welch's two-sample $t$-test. SD; standard deviation

Similarly, we represented smoothed mean curves of biochemical parameters that were statistically different between males and females. As shown in Figure 2, the bandwidth represents a $95 \%$ confidence interval. Therefore, there is a 0.95 probability that the true regression line for the population lies within the confidence interval. 


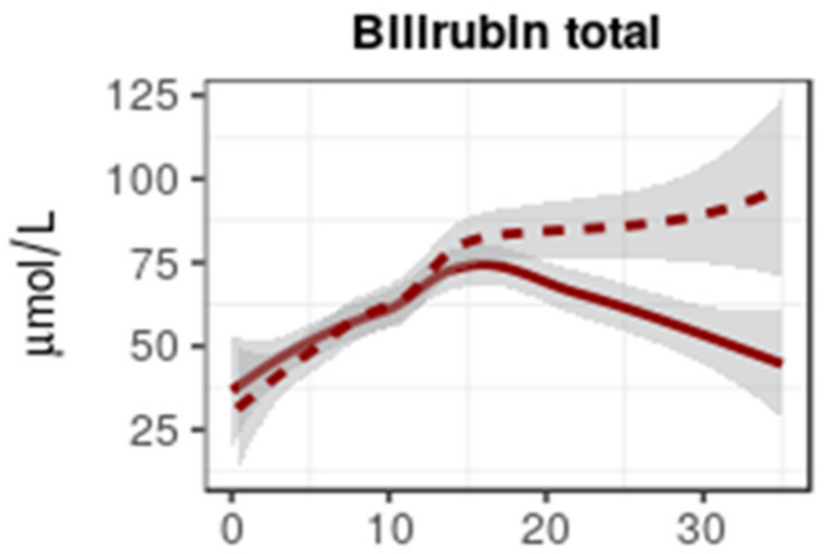

ALP

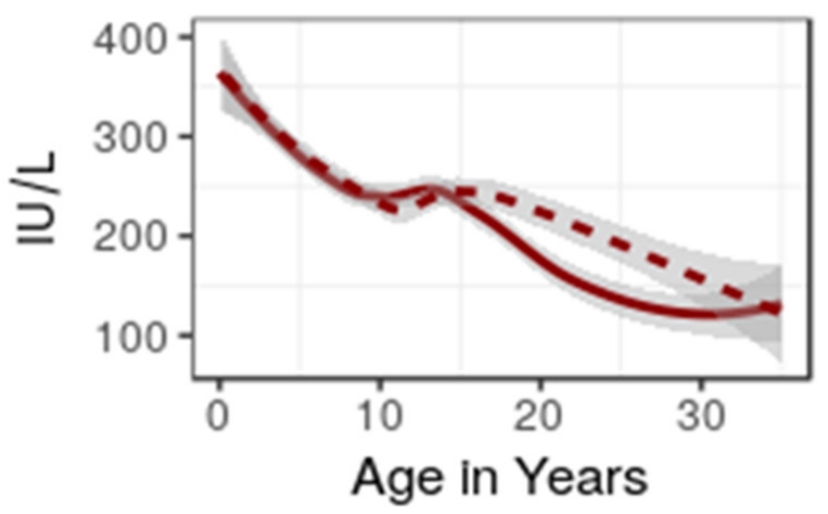

AST

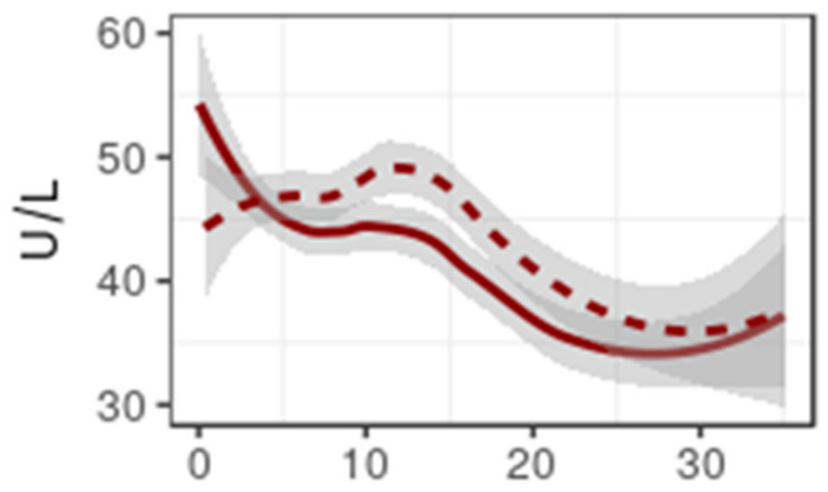

Creatinine

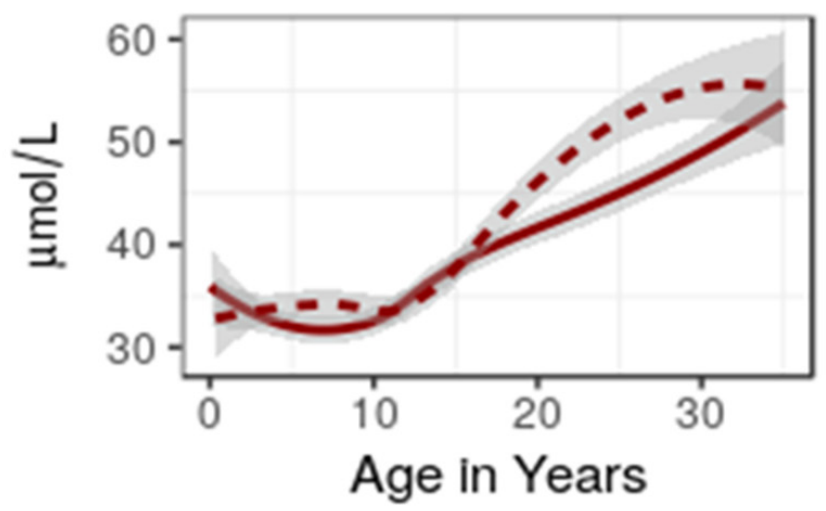

Figure 2. Smoothed mean curves for selected biochemical parameters by sex. Solid lines denote the mean values for females and dashed lines denote the mean values for males.

\subsection{Laboratory Parameters in SCD Compared against the General Population}

Table A1 compares values in SCD and that of the general Tanzanian population published elsewhere $[16,20,21]$. Individuals with SCD had significantly lower reference values for hemoglobin, $\mathrm{MCH}$, and lymphocytes. In comparison, the values for platelets (except infants' group), WBC, bilirubin direct, bilirubin total, and AST were significantly higher when compared to that of the general population (almost $p<0.0001$ in each case). The MCHC, neutrophils, platelets, and ALP in infants had estimated ranges comparable to the general population. Children with SCD below five years old had significantly higher MCV and creatinine reference values than those of the general population, but this changed for the older age groups where reference values from the general population were higher compared to those of individuals with SCD.

\section{Discussion}

Understanding reference ranges for laboratory parameters is critical to the focused care of SCD patients. This study is the first to report reference values for hematological and biochemical parameters for patients with SCD at steady state, categorized by age groups and by sex, in Tanzania. Most laboratory parameters (platelets, white blood cells, bilirubin direct, bilirubin total, aspartate aminotransferase, and alkaline phosphatase) were found to have higher values when compared to that of the general population, which also differed across the age groups. The reference ranges for SCD patients more or less similar between males and females.

The observed steady state hemoglobin concentration of 7.2-7.3 g/ $\mathrm{dL}$ in children with SCD under five years of age was higher than the 6.3-6.9 g/dL reported elsewhere $[9,14,22]$; but lower than 8.1-9.3 g/dL, which was reported in the cooperative study involving 
patients with SCD conducted in Western countries [23]. These observations of differences in hemoglobin may be due to different factors, such as quality of care in children with SCD malaria infection, nutritional deficiency [24], and parasitic infections [25], which are more common in the least developed countries [26].

Moreover, the lower steady state hemoglobin concentration in SCD patients when compared to the general population could be explained by the continual hemolysis of the red cells with their consequent shortened survival in individuals with SCD [15]. The mean hemoglobin, $\mathrm{HbF}, \mathrm{MCH}, \mathrm{RBC}, \mathrm{MCV}$, RDW, eosinophils, bilirubin total, ALP, and creatinine were different between females and males, with most deviations happening after the age of 15 years. This is likely due to the start of the menstruation period, which has also been associated with changes in the levels of these parameters in female macaques [27].

We found significantly higher MCV values in children with SCD younger than five years of age compared to those of the general population. These higher values were in agreement with findings from another study [13]. It was reported that higher MCV in children is largely due to folate deficiency [28]. An insufficiency of vitamin B9 (resulting from not eating enough foods such as leafy green vegetables, beans, and grains) may be worse, especially in under-fives who are more dependent and may have lower compliance with supplementary folate [28].

Our study reported higher platelet counts among individuals with SCD compared to the values reported by the previous study [8]. Furthermore, there was a significant difference in platelet levels in all age groups, except among infants, when the values were compared between individuals with SCD and the general population. After four years of life, platelet counts were gradually elevated in individuals with SCD but declined in the general population. The advancement of platelet count in patients with SCD could be due to deficient splenic pooling of young, active platelets due to hyposplenism [29].

The mean WBC counts were higher among patients with SCD compared to the general population. A previous study suggested that reactions to commonly used medication and recurrent infections are factors that could increase the number of white cells in individuals with SCD [30]. Additionally, elevated WBC counts were shown to occur in children with SCD during bacterial infections and vaso-occlusive crises [31]. Based on these findings, we believe that periodic evaluation of SCD patients through physical examination will help identify those with high WBC so that appropriate measures can be taken to moderate causes related to high WBC.

We reported normal alkaline phosphatase levels in infants while other patients with SCD had shown a significant increase compared to the general population. As documented elsewhere, raised plasma levels of alkaline phosphatase in patients with SCD could result from persistent vaso-occlusive crises involving the bones. The observed elevation of alkaline phosphatase was above the expected rise in children who are still growing [32].

Creatinine levels were considerably lower in patients with SCD after four years of age. The low creatinine values in patients with SCD were in agreement with those reported in other studies $[33,34]$. It was reported that the decrease of creatinine in patients with SCD was caused by smaller physical stature [35]. This finding is consistent with that of another study which investigated uric acid, urea, and creatinine levels in patients with SCD, which reported that the decline of creatinine resulted from the reduced muscle mass [34].

\section{Materials and Methods}

\subsection{Study Design and Setting}

This was a prospective cohort study to establish the hematological and biochemical reference values of individuals with SCD at steady state. The study utilized the Muhimbili Sickle Cohort data from the Muhimbili National Hospital, Tanzania [36]. The Muhimbili Sickle Cohort, established in 2004, had enrolled over 5000 patients with SCD. The patients enrolled in the cohort came from different geographical regions of the country, with varying prevalences of SCD [36]. Grounded on ethnic descent, almost all the patients were homozygous for the Bantu or Central African Republic haplotype [36,37]. Common outpatient 
services offered to patients at SCD clinics included routine physical and hematological monitoring, folic acid supplementation, penicillin prophylaxis for children below six years, and health education. The use of hydroxyurea was not common and none of the patients in this study were using this medication. Patients requiring further care were channeled to appropriate inpatient departments. Other services such as pneumococcal vaccines (since 2013) were provided to patients free of charge through appropriate national programs.

\subsection{Patients}

Hematological and biochemical data were collected longitudinally from 2004 to 2015. Data on 4422 patients with confirmed homozygous SCD (HbSS, via hemoglobin electrophoresis, isoelectric focusing, or high-performance liquid chromatography) and 15,461 visits were available for analysis. Individuals with all the other hemoglobin genotypes were excluded. Patients were categorized into pediatrics (infants, children, and adolescents) and adult groups. The age groups included $<1$ year (infants); $1-4$ years, and 5-11 years (children); 12-17 years (adolescents); and those aged 18 years and above (adults). Only the visiting records of patients at steady state were included in the current study. Steady state was defined as the point in time where the SCD patient was in good health (absence of clinical events) and had not received treatments other than routine penicillin prophylaxis and folate supplements. Furthermore, a patient was not on hydroxyurea, had no history of blood transfusion during the last four months, and had no history of acute pain that required treatment in the emergency department within one month before the observation $[10,38]$.

\subsection{Hematological and Biochemical Parameters}

Twenty-two laboratory parameters were used in this study. Among those, 16 were hematological parameters including hemoglobin $(\mathrm{Hb})$ count, fetal hemoglobin $(\mathrm{HbF})$, mean corpuscular hemoglobin $(\mathrm{MCH})$, mean corpuscular hemoglobin concentration (MCHC), red blood cell (RBC) count, mean corpuscular volume (MCV), red cell distribution width (RDW), reticulocyte count, platelet count, and mean platelet volume (MPV), as well as white blood cells (WBC), neutrophils, lymphocytes, eosinophils, monocytes, and basophils. The other six were biochemical parameters that included bilirubin total, direct bilirubin, aspartate aminotransferase (AST), alkaline phosphatase (ALP), lactate dehydrogenase $(\mathrm{LDH})$, and creatinine. The hematological parameters were assessed using an automated hematology analyzer (Sysmex XT 2000i Kobe, Hyogo, Japan). The biochemical parameters were assessed using Roche Cobas integral 400 plus. All laboratory procedures were done using standard protocols, including appropriate controls.

\subsection{Statistical Analysis}

We assessed data for outliers using box-and-whisker plots. Values below the lowest and above the highest observations were considered outliers and excluded from the analysis. Repeated measurements from individuals were collapsed by calculating the mean value for everyone within the age range using an aggregate function in R. Variables were tested for normality of distribution using diagnostic plots (i.e., Q-Q plots, boxplots, and histograms), the Shapiro-Wilk test, and the Anderson-Darling test. Some variables were transformed using log and square root for those that didn't pass the normality tests. Upper and lower normal values were determined using the mean plus or minus 1.96 standard deviations [39]. The sample population was grouped by sex for each age category. Mean differences between males and females and against the general population were evaluated using the $t$-test and Mann-Whitney U test, respectively. Loess regression was used to smooth the curves for all parameters, which were identified to be significantly different. All ages above 35 were rounded to 35 years to restrict fewer observations (only $1.46 \%$ of the study population) in higher ages, thus removing potential bias in the approximation of the means due to wide bandwidths above 35 years of age. A $p$-value of less than 0.05 was considered significant. 


\section{Conclusions}

In conclusion, our study was the first to report steady state hematological and biochemical reference values for SCD for different age groups (infants, children, adolescents, and adults) separated by sex. We reported an increase in the means of $\mathrm{MCH}, \mathrm{MCV}$, and neutrophils with significantly higher values in females. Other parameters such as $\mathrm{HbF}$, RDW, WBC, and ALP significantly decreased with age in males and females. Moreover, we observed the means of some parameters such as hemoglobin, RBC, platelet, MPV, AST, eosinophils, total bilirubin, and creatinine fluctuate over time between males and females. These data provide useful reference ranges that can be used in monitoring the status and management of patients with SCD in Tanzania and other populations sharing similar environments, particularly in Sub-Saharan Africa.

\section{Limitations}

Although the sample size was large in our study, the number of males and female patients in the infants' group was less than 120, as recommended elsewhere [40,41]. This may be the reason for the failure of a considerable number of parameters in this age group to attain the significance level. Furthermore, the interpretation of the laboratory results may have been limited when the variation of a specific group was minimal. Therefore, the criterion of partitioning the population reference ranges into subgroups (males and females in our case) using a significant difference criterion may seem insufficient compared to utilizing the clinical and physiological approach $[40,41]$.

Author Contributions: B.P.M., R.Z.S. and J.M. (Julie Makani) designed the research study; B.P.M., R.Z.S., A.D.F. and E.B. designed the methodology section; A.D.F., R.Z.S., J.M. (Josephine Mgaya), D.S., F.T., U.M., E.B., S.N., J.M. (Julie Makani) and B.P.M. collected and curated the data; A.D.F. and B.P.M. performed data analysis; A.D.F., R.Z.S., B.P.M., E.B. and J.M. (Julie Makani) interpreted the results; A.D.F. wrote the original draft of the manuscript; A.D.F., R.Z.S., J.M. (Josephine Mgaya), D.S., F.T., U.M., E.B., S.N., J.M. (Julie Makani) and B.P.M. reviewed the manuscript; B.P.M., R.Z.S. and J.M. (Julie Makani) provided project administration and supervision. All authors have read and agreed to the final draft. All authors have read and agreed to the published version of the manuscript.

Funding: No funding was provided for this study. Original data collection was funded by the Wellcome Trust, UK (Fellowship Julie Makani 072064, 093727; Project grant 080025; Strategic award 084538; Principal Wellcome Trust Fellowship R W Snow 103602 and Government of United Republic of Tanzania.

Institutional Review Board Statement: The study was conducted according to the guidelines of the Muhimbili National Hospital (MNH) and approved by Muhimbili University of Health and Allied Science (MUHAS) ethical committee (MU/RP/AEC/VOL XI/33).

Informed Consent Statement: During data collection, informed consent was obtained from all SCD patients or parents/guardians in the case of children and adolescents (below 18 years).

Data Availability Statement: The Muhimbili data sharing policy is not finalized. The data used to support the findings of this study are available from the institute upon reasonable request.

Acknowledgments: We acknowledge the support from all SCD patients and the Muhimbili sickle cell programme staff. A.D.F. thanks the African Institute for Mathematical Sciences-GlaxoSmithKline (AIMS-GSK) for the internship financial supports.

Conflicts of Interest: The authors declare that they have no competing interest. 


\section{Appendix A}

Table A1. Medians (95\% reference ranges) in selected laboratory parameters for SCD at steady states compared to published population data in Tanzania.

\begin{tabular}{|c|c|c|c|c|}
\hline Analytes (Unit) & $\begin{array}{l}\text { Age Group in } \\
\text { Years } \\
\text { (sex) }\end{array}$ & $\begin{array}{c}\text { SCD Patients } \\
\text { Median } \\
(2.5-97.5 \%)\end{array}$ & $\begin{array}{c}\text { General } \\
\text { Population } \\
\text { Median } \\
(2.5-97.5 \%) \\
\end{array}$ & $p$-Values * \\
\hline \multirow{8}{*}{$\begin{array}{l}\text { Hemoglobin } \\
\text { (g/dL) }\end{array}$} & $<1$ & $7.3(4.6-9.5)$ & $10.7(8.1-13.2)^{\dagger}$ & $<0.0001$ \\
\hline & $1-4$ & $7.3(4.5-9.7)$ & $11.3(8.1-13.9)^{\dagger}$ & $<0.0001$ \\
\hline & 5-11 & $7.3(4.9-9.4)$ & $12.6(10.3-14.7)^{\dagger}$ & $<0.0001$ \\
\hline & $12-17(\mathrm{~F})$ & $7.6(5.3-9.7)$ & $13.0(10.0-14.9)^{\dagger}$ & $<0.0001$ \\
\hline & $12-17(\mathrm{M})$ & $7.4(5.0-9.8)$ & $13.7(10.8-17.0)^{\dagger}$ & $<0.0001$ \\
\hline & $\geq 18(\mathrm{~F})$ & $7.6(5.1-10.0)$ & $13.5(11.1-15.7) \ddagger$ & $<0.0001$ \\
\hline & $\geq 18(\mathrm{M})$ & $8.3(4.7-11.0)$ & $15.4(13.7-17.7)^{\ddagger}$ & $<0.0001$ \\
\hline & $\geq 18(\mathrm{M} \& \mathrm{~F})$ & $7.9(4.8-10.6)$ & $14.7(11.7-17.2) \ddagger$ & $<0.0001$ \\
\hline \multirow{4}{*}{ MCH (pg) } & - & - & - & - \\
\hline & $\geq 18(\mathrm{~F})$ & $28.0(19.9-34.2)$ & $30.0(23.1-33.2) \ddagger$ & $<0.0001$ \\
\hline & $\geq 18(\mathrm{M})$ & $26.6(19.9-33.1)$ & $29.3(24.2-33.1) \ddagger$ & $<0.0001$ \\
\hline & $\geq 18(\mathrm{M} \& \mathrm{~F})$ & $27.5(19.9-34.0)$ & $29.7(23.6-33.1)^{\ddagger}$ & $<0.0001$ \\
\hline \multirow{4}{*}{ MCHC (g/dL) } & - & - & - & - \\
\hline & $\geq 18(\mathrm{~F})$ & $32.8(29.9-36.1)$ & $33.3(30.6-35.1) \ddagger$ & $<0.0001$ \\
\hline & $\geq 18(\mathrm{M})$ & $32.6(30.0-36.5)$ & $32.7(30.4-34.8) \ddagger$ & 0.9239 \\
\hline & $\geq 18(\mathrm{M} \& \mathrm{~F})$ & $32.8(30.0-36.3)$ & $33.0(30.6-34.9) \ddagger$ & 0.9856 \\
\hline \multirow{8}{*}{ MCV (fL) } & $<1$ & $74.0(53.4-91.7)$ & $71.0(53.3-96.6)^{\dagger}$ & $<0.0001$ \\
\hline & $1-4$ & 79.0 (59.7-95.3) & $75.0(54.7-91.6)^{\dagger}$ & $<0.0001$ \\
\hline & 5-11 & $80.0(60.5-98.0)$ & $82.5(66.0-90.0)^{\dagger}$ & $<0.0001$ \\
\hline & $12-17(\mathrm{~F})$ & $82.0(64.3-99.1)$ & $84.9(62.2-94.5)^{\dagger}$ & $<0.0001$ \\
\hline & $12-17(\mathrm{M})$ & $79.0(63.4-93.5)$ & $82.0(63.2-91.0)^{\dagger}$ & $<0.0001$ \\
\hline & $\geq 18(\mathrm{~F})$ & $84.4(64.0-102.8)$ & $89.5(77.7-97.9) \ddagger$ & $<0.0001$ \\
\hline & $\geq 18(\mathrm{M})$ & 81.7 (62.8-99.2) & $89.3(76.4-98.8) \ddagger$ & $<0.0001$ \\
\hline & $\geq 18(\mathrm{M} \& \mathrm{~F})$ & $83.3(63.0-101.4)$ & $89.4(77.6-98.1) \ddagger$ & $<0.0001$ \\
\hline \multirow{8}{*}{$\begin{array}{l}\text { Platelet Count } \\
\qquad\left(\times 10^{9} / \mathrm{L}\right)\end{array}$} & $<1$ & $399(135-724)$ & $384(25-708)^{\dagger}$ & 0.2923 \\
\hline & $1-4$ & $376(104-758)$ & $359(79-650)^{\dagger}$ & 0.0087 \\
\hline & $5-11$ & $432(126-762)$ & $320(94-530)^{+}$ & $<0.0001$ \\
\hline & $12-17(\mathrm{~F})$ & $450(132-771)$ & $282(107-482)^{\dagger}$ & $<0.0001$ \\
\hline & $12-17(\mathrm{M})$ & 445 (179-808) & $271(119-458)^{+}$ & $<0.0001$ \\
\hline & $\geq 18(\mathrm{~F})$ & $448(143-738)$ & $271(151-425)^{\ddagger}$ & $<0.0001$ \\
\hline & $\geq 18(\mathrm{M})$ & 435 (179-777) & $224(147-356) \ddagger$ & $<0.0001$ \\
\hline & $\geq 18(\mathrm{M} \& \mathrm{~F})$ & $439(148-765)$ & $244(150-359) \ddagger$ & $<0.0001$ \\
\hline \multirow{5}{*}{$\begin{array}{l}\text { WBC count } \\
\left(\times 10^{9} / \mathrm{L}\right)\end{array}$} & $<1$ & $13.8(7.8-33.7)$ & $9.2(5.0-17.3)^{\dagger}$ & $<0.0001$ \\
\hline & $1-4$ & $17.2(8.2-30.1)$ & $7.7(3.7-13.2)^{\dagger}$ & $<0.0001$ \\
\hline & $5-11$ & $14.7(7.7-25.3)$ & $5.6(3.7-9.1)^{\dagger}$ & $<0.0001$ \\
\hline & $12-17$ & $12.6(6.8-21.1)$ & $5.3(3.2-10.3)^{\dagger}$ & $<0.0001$ \\
\hline & $\geq 18$ & - & - & - \\
\hline \multirow{4}{*}{$\begin{array}{c}\text { Lymphocytes } \\
(\%)\end{array}$} & - & - & - & - \\
\hline & $\geq 18(\mathrm{~F})$ & $37.3(19.7-53.1)$ & $40.4(20.9-56.7)^{\ddagger}$ & $<0.0001$ \\
\hline & $\geq 18(\mathrm{M})$ & $36.8(16.9-54.2)$ & $40.8(20.8-57.3) \ddagger$ & $<0.0001$ \\
\hline & $\geq 18(\mathrm{M} \& \mathrm{~F})$ & $37.1(18.2-54.6)$ & $40.4(20.8-56.7) \ddagger$ & $<0.0001$ \\
\hline \multirow{4}{*}{$\begin{array}{c}\text { Neutrophils } \\
(\%)\end{array}$} & - & - & - & - \\
\hline & $\geq 18(\mathrm{~F})$ & $48.6(30.4-67.3)$ & $48.4(32.5-71.3) \ddagger$ & 0.7035 \\
\hline & $\geq 18(\mathrm{M})$ & $46.8(26.8-69.7)$ & $47.3(31.7-69.3)^{\ddagger}$ & 0.4763 \\
\hline & $\geq 18(\mathrm{M} \& \mathrm{~F})$ & $47.6(28.5-69.6)$ & $48.1(32.0-69.1)^{\ddagger}$ & 0.6826 \\
\hline \multirow{4}{*}{$\begin{array}{l}\text { Bilirubin Direct } \\
\quad(\mu \mathrm{mol} / \mathrm{L})\end{array}$} & - & - & - & - \\
\hline & $\geq 18(\mathrm{~F})$ & $8.1(0.0-23.4)$ & $1.81(0.70-5.83) \ddagger$ & $<0.0001$ \\
\hline & $\geq 18(\mathrm{M})$ & $7.5(0.0-32.9)$ & $2.53(0.93-8.43) \ddagger$ & $<0.0001$ \\
\hline & $\geq 18(\mathrm{M} \& \mathrm{~F})$ & $7.8(0.0-29.3)$ & $2.21(0.72-8.22) \ddagger$ & $<0.0001$ \\
\hline
\end{tabular}


Table A1. Cont.

\begin{tabular}{|c|c|c|c|c|}
\hline Analytes (Unit) & $\begin{array}{c}\text { Age Group in } \\
\text { Years } \\
\text { (sex) }\end{array}$ & $\begin{array}{c}\text { SCD Patients } \\
\text { Median } \\
(2.5-97.5 \%)\end{array}$ & $\begin{array}{l}\text { General Population } \\
\text { Median }(2.5-97.5 \%)\end{array}$ & $p$-Values * \\
\hline \multirow{6}{*}{$\begin{array}{l}\text { Bilirubin Total } \\
(\mu \mathrm{mol} / \mathrm{L})\end{array}$} & $<1$ & $32.2(10.4-90.5)$ & $4(2-19){ }^{P}$ & $<0.0001$ \\
\hline & $1-4$ & $36.9(7.8-132.3)$ & $4(2-9)^{\mathbb{P}}$ & $<0.0001$ \\
\hline & $12-17(\mathrm{M})$ & $57.8(10.5-295.2)$ & $6(2-15){ }^{\mathbb{P}}$ & $<0.0001$ \\
\hline & $\geq 18(\mathrm{~F})$ & $45.5(10.2-170.9)$ & $13.9(6.0-42.0) \ddagger$ & $<0.0001$ \\
\hline & $\geq 18(\mathrm{M})$ & $52.6(12.9-251.8)$ & $10.0(4.5-31.3) \ddagger$ & $<0.0001$ \\
\hline & $\geq 18(\mathrm{M} \& \mathrm{~F})$ & $48.6(10.9-216.0)$ & $11.5(5.2-41.0) \ddagger$ & $<0.0001$ \\
\hline \multirow{8}{*}{ AST (U/L) } & $<1$ & $46.0(11.8-101.2)$ & $38(26-65)$ & 0.0057 \\
\hline & $1-4$ & $43.0(24.4-89.3)$ & $36(27-55)^{P}$ & $<0.0001$ \\
\hline & $5-11$ & $43.5(24.0-86.8)$ & $31(21-51)^{\mathbb{P}}$ & $<0.0001$ \\
\hline & $12-17(\mathrm{~F})$ & $38.0(20.0-86.2)$ & $23(17-36)^{P}$ & $<0.0001$ \\
\hline & $12-17(\mathrm{M})$ & $44.0(22.5-90.0)$ & $27(19-42)^{P}$ & $<0.0001$ \\
\hline & $\geq 18(\mathrm{~F})$ & $32.0(16.7-66.0)$ & $20.1(13.5-35.2) \ddagger$ & $<0.0001$ \\
\hline & $\geq 18(\mathrm{M})$ & $31.0(18.0-78.9)$ & $28.2(15.2-53.4) \ddagger$ & $<0.0001$ \\
\hline & $\geq 18(\mathrm{M} \& \mathrm{~F})$ & $31.0(18.0-61.8)$ & $22.8(14.3-48.1)^{\ddagger}$ & $<0.0001$ \\
\hline \multirow{8}{*}{ ALP (IU/L) } & $<1$ & $322(53-586)$ & $307(164-589){ }^{P}$ & 0.1998 \\
\hline & $1-4$ & $288(120-600)$ & $253(153-410)^{\mathbb{P}}$ & $<0.0001$ \\
\hline & $5-11$ & $237(110-468)$ & $278(174-460)^{\mathbb{P}}$ & $<0.0001$ \\
\hline & $12-17(\mathrm{~F})$ & $216(71-556)$ & $191(68-498)^{\mathbb{P}}$ & $<0.0001$ \\
\hline & $12-17(\mathrm{M})$ & $229(122-488)$ & $302(124-537){ }^{\mathbb{P}}$ & $<0.0001$ \\
\hline & $\geq 18(\mathrm{~F})$ & $115(46-310)$ & $85.0(45.4-170.4) \ddagger$ & $<0.0001$ \\
\hline & $\geq 18(\mathrm{M})$ & $153(59-408)$ & $75.4(45.3-155.0) \ddagger$ & $<0.0001$ \\
\hline & $\geq 18(\mathrm{M} \& \mathrm{~F})$ & $123(47-396)$ & $80.0(45.6-158.4) \ddagger$ & $<0.0001$ \\
\hline \multirow{8}{*}{$\begin{array}{c}\text { Creatinine } \\
(\mu \mathrm{mol} / \mathrm{L})\end{array}$} & $<1$ & $32.5(13.2-57.1)$ & $18(12-28) P^{P}$ & $<0.0001$ \\
\hline & $1-4$ & $32.0(20.0-56.0)$ & $24(15-50){ }^{\mathbb{P}}$ & $<0.0001$ \\
\hline & $5-11$ & $32.0(18.0-56.0)$ & $36(24-49)^{\mathbb{P}}$ & $<0.0001$ \\
\hline & $12-17(\mathrm{~F})$ & $35.0(19.1-68.0)$ & $45(32-64)^{P}$ & $<0.0001$ \\
\hline & $12-17(\mathrm{M})$ & $34.0(18.0-69.1)$ & $48(36-80)^{P}$ & $<0.0001$ \\
\hline & $\geq 18(\mathrm{~F})$ & $42.5(25.0-86.0)$ & $56(40-81) \ddagger$ & $<0.0001$ \\
\hline & $\geq 8(\mathrm{M})$ & $51.0(26.3-83.4)$ & $69(48-96) \ddagger$ & $<0.0001$ \\
\hline & $\geq 18(\mathrm{M} \& \mathrm{~F})$ & $44.7(25.0-85.3)$ & $62(42-90) \ddagger$ & $<0.0001$ \\
\hline
\end{tabular}

* Mann-Whitney U test for significance difference. ${ }^{\dagger}$ Obtained from [16]; ${ }^{\mathbb{P}}$ from [20] and ${ }^{\ddagger}$ from [21]. In [16,20] age ranges were listed as; $<12$ months, 1 to $<5$ year, 5 to $<13$ years, 13 to $<18$ years (M), 13 to $<18$ years (F). In [21] age range is 19 to 48 years.

\section{References}

1. Modell, B.; Darlison, M. Global epidemiology of haemoglobin disorders and derived service indicators. Bull. World Health Organ. 2008, 86, 480-487. [CrossRef]

2. Piel, F.B.; Hay, S.I.; Gupta, S.; Weatherall, D.J.; Williams, T.N. Global Burden of Sickle Cell Anaemia in Children under Five, 2010-2050: Modelling Based on Demographics, Excess Mortality, and Interventions. PLoS Med. 2013, 10, e1001484. [CrossRef]

3. Makani, J.; Komba, A.N.; Cox, S.; Oruo, J.; Mwamtemi, K.; Kitundu, J.; Magesa, P.; Rwezaula, S.; Meda, E.; Mgaya, J.; et al. Malaria in patients with sickle cell anemia: Burden, risk factors, and outcome at the outpatient clinic and during hospitalization. Blood 2010, 115, 215-220. [CrossRef] [PubMed]

4. Makani, J.; Cox, S.; Soka, D.; Komba, A.N.; Oruo, J.; Mwamtemi, H.; Magesa, P.; Rwezaula, S.; Meda, E.; Mgaya, J.; et al. Mortality in sickle cell anemia in Africa: A prospective cohort study in Tanzania. PLoS ONE 2011, 6, e14699. [CrossRef]

5. Tluway, F.; Makani, J. Sickle cell disease in Africa: An overview of the integrated approach to health, research, education and advocacy in Tanzania, 2004-2016. Br. J. Haematol. 2017, 177, 919-929. [CrossRef] [PubMed]

6. Bhatia, M.; Walters, M.C. Hematopoietic cell transplantation for thalassemia and sickle cell disease: Past, present and future. Bone Marrow Transplant. 2008, 41, 109-117. [CrossRef] [PubMed]

7. Vermylen, C.; Cornu, G.; Ferster, A.; Brichard, B.; Ninane, J.; Ferrant, A.; Zenebergh, A.; Maes, P.; Dhooge, C.; Benoit, Y.; et al. Haematopoietic stem cell transplantation for sickle cell anaemia: The first 50 patients transplanted in Belgium. Bone Marrow Transplant. 1998, 22, 1-6. [CrossRef] 
8. Akinbami, A.; Dosunmu, A.; Adediran, A.; Oshinaike, O.; Adebola, P.; Arogundade, O. Haematological values in homozygous sickle cell disease in steady state and haemoglobin phenotypes AA controls in Lagos, Nigeria. BMC Res. Notes $2012,5,396$. [CrossRef]

9. Iheanacho, O. Haematological Parameters of Adult and Paediatric Subjects with Sickle Cell Disease in Steady State, in Benin City, Nigeria. Int. Blood Res. Rev. 2015, 3, 171-177. [CrossRef]

10. Ballas, S.K.; Smith, E.D. Red blood cell changes during the evolution of the sickle cell painful crisis. Blood 1992, 79, 2154-2163. [CrossRef]

11. Okpala, I.; Tawil, A. Management of pain in sickle-cell disease. J. R. Soc. Med. 2002, 95, 456-458. [CrossRef]

12. Powars, D.R.; Schroeder, W.A.; Weiss, J.N.; Chan, L.S.; Azen, S.P. Lack of influence of fetal hemoglobin levels or erythrocyte indices on the severity of sickle cell anemia. J. Clin. Investig. 1980, 65, 732-740. [CrossRef]

13. Omoti, C.E. Haematological values in sickle cell anaemia in steady state and during vaso-occlusive crisis in Benin City, Nigeria. Ann. Afr. Med. 2005, 4, 62-67.

14. Akodu, S.O.; Njokanma, O.F.; AdeoluKehinde, O. Erythrocyte indices in Pre-School Nigerian children with sickle cell anaemia in steady state. Int. J. Hematol.-Oncol. Stem Cell Res. 2015, 9, 5-9.

15. Emmanuelchide, O.; Charle, O.; Uchenna, O. Hematological parameters in association with outcomes in sickle cell anemia patients. Indian J. Med. Sci. 2011, 65, 393. [CrossRef] [PubMed]

16. Buchanan, A.M.; Muro, F.J.; Gratz, J.; Crump, J.A.; Musyoka, A.M.; Sichangi, M.W.; Morrissey, A.B.; M'Rimberia, J.K.; Njau, B.N.; Msuya, L.J.; et al. Establishment of haematological and immunological reference values for healthy Tanzanian children in Kilimanjaro Region. Trop. Med. Int. Health 2010, 15, 1011-1021. [CrossRef]

17. Lugada, E.S.; Mermin, J.; Kaharuza, F.; Ulvestad, E.; Were, W.; Langeland, N.; Asjo, B.; Malamba, S.; Downing, R. Population-based hematologic and immunologic reference values for a healthy Ugandan population. Clin. Diagn. Lab. Immunol. 2004, 11, 29-34. [CrossRef]

18. Quintó, L.; Aponte, J.J.; Sacarlal, J.; Espasa, M.; Aide, P.; Mandomando, I.; Guinovart, C.; Macete, E.; Navia, M.M.; Thompson, R.; et al. Haematological and biochemical indices in young African children: In search of reference intervals. Trop. Med. Int. Health 2006, 11, 1741-1748. [CrossRef] [PubMed]

19. Katayev, A.; Balciza, C.; Seccombe, D.W. Establishing reference intervals for clinical laboratory test results: Is there a better way? Am. J. Clin. Pathol. 2010, 133, 180-186. [CrossRef] [PubMed]

20. Buchanan, A.M.; Fiorillo, S.P.; Omondi, M.W.; Cunningham, C.K.; Crump, J.A. Establishment of biochemistry reference values for healthy Tanzanian infants, children and adolescents in Kilimanjaro Region. Trop. Med. Int. Health 2015, 20, 1569-1577. [CrossRef]

21. Saathoff, E.; Schneider, P.; Kleinfeldt, V.; Geis, S.; Haule, D.; Maboko, L.; Samky, E.; de Souza, M.; Robb, M.; Hoelscher, M. Laboratory reference values for healthy adults from southern Tanzania. Trop. Med. Int. Health 2008, 13, 612-625. [CrossRef]

22. Abbas, M. Haematological parameters in Sudanese children with sickle cell disease. Am. J. Res. Com. 2014, 2, 20-32.

23. Brown, A.K.; Sleeper, L.A.; Miller, S.T.; Pegelow, C.H. Reference Values and. Arch. Pediatr. Adolesc. Med. 2012, 148, 796-804. [CrossRef] [PubMed]

24. Reed, J.D.; Redding-Lallinger, R.; Orringer, E.P. Nutrition and sickle cell disease. Am. J. Hematol. 1987, 24, 441-455. [CrossRef]

25. Henrici, R.C.; Sautter, C.L.; Bond, C.; Opoka, R.O.; Namazzi, R.; Datta, D.; Ware, R.E.; Conroy, A.L.; John, C.C. Decreased parasite burden and altered host response in children with sickle cell anemia and severe anemia with malaria. Blood Adv. 2021, 5, 4710-4720. [CrossRef]

26. Desai, M.R.; Terlouw, D.J.; Kwena, A.M.; Phillips-Howard, P.A.; Kariuki, S.K.; Wannemuehler, K.A.; Odhacha, A.; Hawley, W.A.; Shi, Y.P.; Nahlen, B.L.; et al. Factors associated with hemoglobin concentrations in pre-school children in western Kenya: Cross-sectional studies. Am. J. Trop. Med. Hyg. 2005, 72, 47-59. [CrossRef]

27. Perigard, C.J.; Parrula, M.C.M.; Larkin, M.H.; Gleason, C.R. Impact of menstruation on select hematology and clinical chemistry variables in cynomolgus macaques. Vet. Clin. Pathol. 2016, 45, 232-243. [CrossRef]

28. Irwin, J.; Kirchner, J. Anemia in children. Nippon Rinsho Jpn. J. Clin. Med. 2008, 66, 544-547.

29. Allen, B.; Schwartz, D. The Splenic Platelet Reservoir. Cell 1972, 40, 678-683.

30. Riley, L.K.; Rupert, J. Evaluation of Patients with Leukocytosis. Am. Fam. Physician 2015, 92, 1004-1011. [PubMed]

31. Buchanan, G.R.; Glader, B.E. Leukocyte Counts in Children with Sickle Cell Disease: Comparative Values in the Steady State, Vaso-occlusive Crisis, and Bacterial Infection. Am. J. Dis. Child. 1978, 132, 396-398. [CrossRef]

32. Kotila, T.; Adedapo, K.; Adedapo, A.; Oluwasola, O.; Fakunle, E.; Brown, B. Liver dysfunction in steady state sickle cell disease. Ann. Hepatol. 2019, 4, 261-263. [CrossRef]

33. Al-Ali, A.K.; Ahmed, M.A.M.; Qaw, F.S.; Al-Fadel Saleh, M.; Al-Bashir, A. Uric Acid, Creatinine and Urea in Normal, Glucose-6Phosphate Dehydrogenase-Deficient and Hb S S Saudi Subjects. Acta Haematol. 1995, 94, 114-116. [CrossRef]

34. Al-naama, L.M.; Al-Sadoon, T.A. Levels of Uric Acid, Urea and Creatinine in Iraqi Children with Sickle Cell Disease. J. -Pak. Med. Assoc. 2000, 50, 98-101. [PubMed]

35. Odonkor, P.O.; Addae, S.K.; Yamamoto, S.; Apatu, R.S. Effect of dietary nitrogen on urinary excretion of non-protein nitrogen in adolescent sickle cell patients. Hum. Nutr. Clin. Nutr. 1984, 38, $23-29$.

36. Makani, J.; Tluway, F.; Makubi, A.; Soka, D.; Nkya, S.; Sangeda, R.; Mgaya, J.; Rwezaula, S.; Kirkham, F.J.; Kindole, C.; et al. A ten year review of the sickle cell program in Muhimbili National Hospital, Tanzania. BMC Hematol. 2018, 18, 33. [CrossRef] [PubMed] 
37. Mtatiro, S.N.; Singh, T.; Rooks, H.; Mgaya, J.; Mariki, H.; Soka, D.; Mmbando, B.; Msaki, E.; Kolder, I.; Thein, S.L.; et al. Genome wide association study of fetal hemoglobin in sickle cell Anemia in Tanzania. PLoS ONE 2014, 9, e111464. [CrossRef]

38. Ballas, S.K. More definitions in sickle cell disease: Steady state v base line data. Am. J. Hematol. 2012, 87, 338. [CrossRef]

39. Bertholf, R.L. Statistical Methods for Establishing and Validating Reference Intervals. Lab. Med. 2006, 37, 306-310. [CrossRef]

40. Reed, A.H.; Henry, R.J.; Mason, W.B. Influence of statistical method used on the resulting estimate of normal range. Clin. Chem. 1971, 17, 275-284. [CrossRef]

41. Harris, E.K.; Wong, E.T.; Shaw, S.T. Statistical criteria for separate reference intervals: Race and gender groups in creatine kinase. Clin. Chem. 1991, 37, 1580-1582. [CrossRef] [PubMed] 